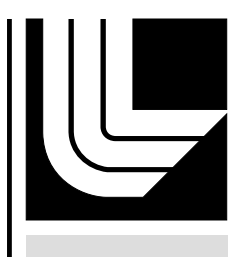

LAW RENCE LIVERMORE N A TION A L LABORATORY

\title{
UCRL-TR-229498
}

\section{RADSRC/Monte Carlo Code Interface Manual}

L. Hiller, J. Gronberg, T. Gosnell, D. M. Wright

March 28, 2007 
This document was prepared as an account of work sponsored by an agency of the United States Government. Neither the United States Government nor the University of California nor any of their employees, makes any warranty, express or implied, or assumes any legal liability or responsibility for the accuracy, completeness, or usefulness of any information, apparatus, product, or process disclosed, or represents that its use would not infringe privately owned rights. Reference herein to any specific commercial product, process, or service by trade name, trademark, manufacturer, or otherwise, does not necessarily constitute or imply its endorsement, recommendation, or favoring by the United States Government or the University of California. The views and opinions of authors expressed herein do not necessarily state or reflect those of the United States Government or the University of California, and shall not be used for advertising or product endorsement purposes.

This work was performed under the auspices of the U.S. Department of Energy by University of California, Lawrence Livermore National Laboratory under Contract W-7405-Eng-48. 
DMC doc 0v1

UCRL-TR-

\title{
RADSRC/Monte Carlo Code Interface Manual
}

\author{
The RADSRC development team \\ Lawrence Livermore National Laboratory \\ June 1, 2004 \\ Revised: June 2, 2004
}

\begin{abstract}
RADSRC is a library for calculating gamma ray distributions. An initial material specification is aged and the daughter isotopes calculated to create the complete spectrum. RADSRC can be linked into, initialized, and called from other programs. This document specifies how to do this in GEANT4, COG and $\operatorname{MCNP}(\mathrm{X})$.
\end{abstract}

\section{Configuring GEANT4 to use RADSRC}

The user will need to have access to a GEANT4 installation. It is assumed that the user can already run a GEANT4 job.

Download the RADSRC library to your computer. It can be found at http://nuclear.llnl.gov. In the src directory type gmake in order to create the libradsrc.a file. It is highly recommended that you determine what compiler the GEANT4 installation is using and modify the RADSRC Makefile to use the same compiler.

The g4 directory in the RADSRC release contains a sample GEANT4 job which accesses the radsrc library and runs a 10kg uranium ball problem. Type source setup to create the RADSRC environment variables needed by the Makefile and the code. If you have GEANT4 installed on your system and the GEANT4 environment variables are set then you should be able to type gmake to create an executable. An executable file called exampleNO1 should be located in the bin directory.

Type bin/[system type]/exampleN01 <example. in to run the program.

The GEANT4 GNU makefile has been modified to link in the RADSRC libraries through the addition of two line:

EXTRALIBS $+=-$ L\$ (RADSRC_HOME) /lib/ -lradsrC

CPPFLAGS $+=-I \$\left(R A D S R C \_H O M E\right) /$ src/libradsrc/

The environment variable RADSRC_HOME is defined in the setup routine along with the variable RADSRC_LEGACYDATA which points to the RADSRC data files.

\section{Calling RADSRC from within GEANT4}

The RADSRC routines are called from within the ExN01PrimaryGeneratorAct ion class which handles event generation for the problem. In the header files we include the RADSRC header files: 
\#include "radsource.h"

\#include "cpp_api.h"

and create some pointers variables that will point to instances of the RADSRC class,

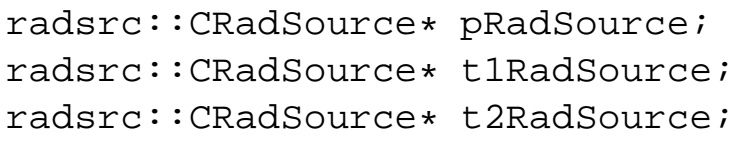

the RADSRC routines live in the namespace radsrc : : in order to prevent conflicts with geant 4 classes.

To create a new instance of RADSRC one calls,

t1RadSource = radsrc: :CApi: : newSource ();

The instance of RADSRC must then be initialized with a problem definition. A number of ways to do that are provided. One way is to create a text file with the problem definition and pass the location of that file to the LoadConfig member function.

t $1 \mathrm{Good}=$ radsrc: :CApi::loadConfig(t1RadSource, (const std: :string) FileName);

If a NULL string is passed the program will look for the environment variable RADSRC_CONF IG to find the input text file.

The text file must be in the form of,

$\begin{array}{ll}\text { U235 } & 90.0 \\ \text { U238 } & 10.0 \\ \text { AGE } & 20.0\end{array}$

where the fraction of each isotope is specified and should add up to $100 \%$. To allow for problems with contamination the fractional sum can be slightly greater than $100 \%$. The last line in the file should be an AGE card with the age given in years.

Additionally, the problem specification can be passed as input lines to the code,

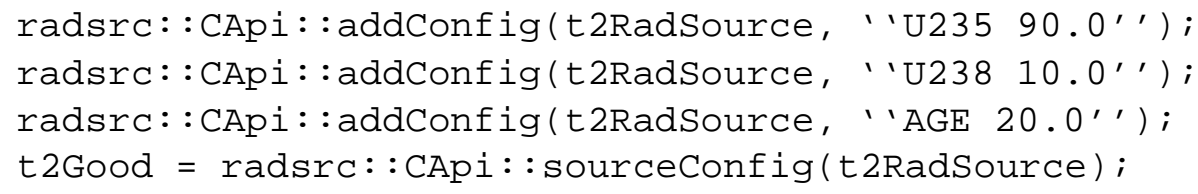

where the addConfig member function accepts text lines of input and the sourceConfig processes the input and performs the RADSRC calculations and setup.

Once the problem is specified the gamma-ray distributions can be sampled with a call to:

G4double energy = radsrc: :CApi: :getPhoton(pRadSource, localran ) * keV;

The function returns an energy in keV. The function localran is a wrapper for the standard GEANT4 random number generator G4Uni formRandom.

The RADSRC problem can also be specified from the GEANT4 command line through the commands defined in the ExN01PrimaryGeneratorMessenger class. They duplicate from the command line what is available in the code.

/radsrc/file'. /problem.in' 
reads the problem definition from the specified file

/radsrc/file' '

will look for the environment variable RADSRC_CONFIG to find the input text file. The complete problem specification can also be passed on the command line by using

/radsrc/input U235 90.0

/radsrc/input U238 10.0

/radsrc/input AGE 20.0

/radsrc/update

The GEANT4 code is set up so that the ExN01PrimaryGeneratorAction class will try to find a RADSRC input text file at instanciation. A constructor function is provided which passes a string which specifies the file location. See exampleN01. cc for an example. That definition can be overridden from the command line. If no problem definition is specified the program will terminate at the first event.

\section{Calling RADSRC from within FORTRAN}

COG and MCNP(X) provide dummy source subroutines called IsoP.F and source.F90, respectively, which can be used to call the RADSRC routines. While RADSRC is native $\mathrm{C}++$, a number of Fortran callable subroutines are provided to allow Fortran code to use the package. All routines names begin with RS in order to prevent accidental conflicts with the Monte Carlo code. Multiple instances of the RADSRC library can be created to model multiple sources. Simply provide a different handle for each case. Inside the subroutine define the variables and functions that the library will use.

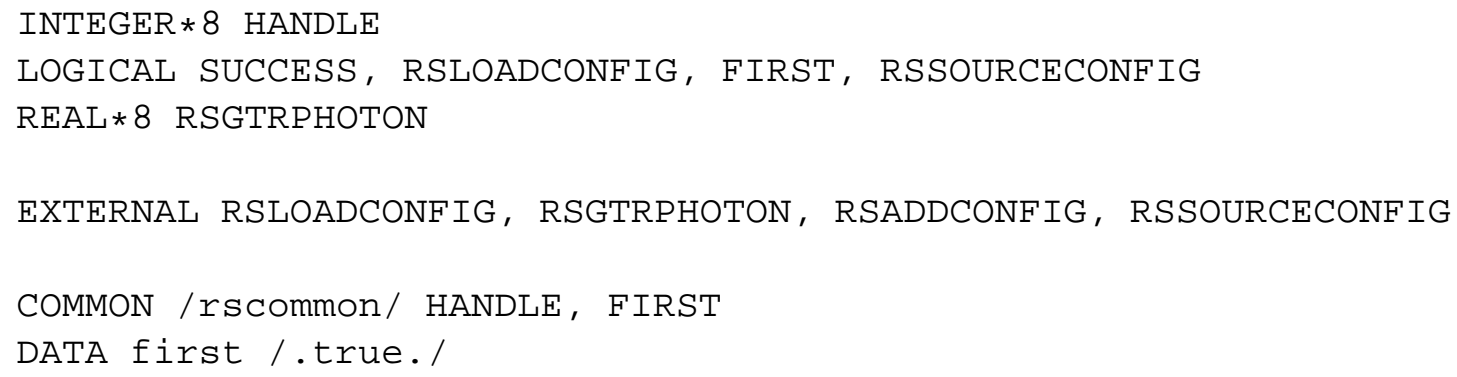

During the first pass through the subroutine an instance of the RADSRC library is created and the problem specified.

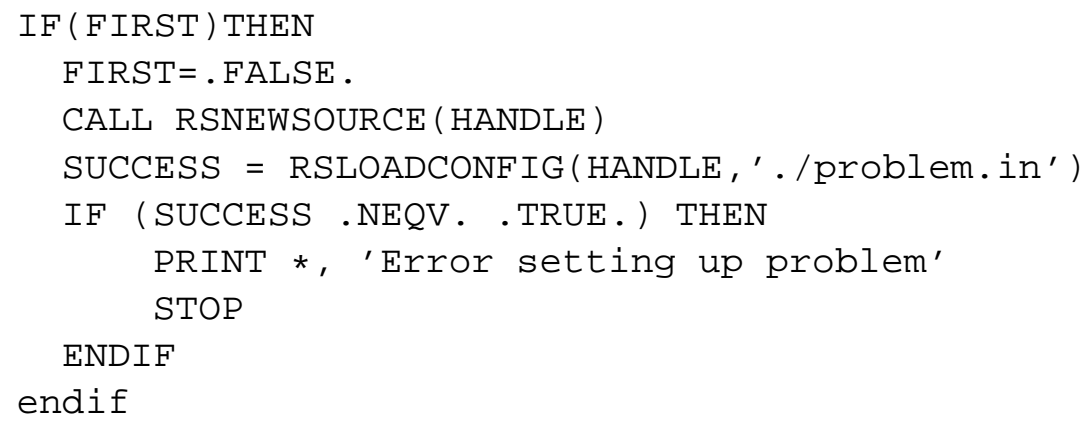


The subroutine RSNEWSOURCE (HANDLE) creates an instance of the RADSRC library and returns a pointer to it. The specification of the problem is held in a text file of the form:

$\begin{array}{ll}\text { U2325 } & 90.0 \\ \text { U238 } & 10.0 \\ \text { AGE } & 20.0\end{array}$

where the fraction of each isotope specified and should add up to 100\%. To allow for problems with contamination the fractional sum can be slightly greater than $100 \%$. The last line in the file should be an AGE card with the age given in years. The subroutine RSLOADCONFIG (HANDLE,' ./problem.in') reads in the specified problem definition file and performs the problem calculations and setup. It returns . FALSE. if there is a problem. If RSLOADCONFIG (HANDLE, ${ }^{\prime}$ ') is specified with a null string then the code will check the environment variable

setenv RADSRC_CONFIG [path to text file with problem specification]

for the location of the input file.

Alternatively, the problem specification can be passed as strings using the RSADDCONF IG and RSSOURCECONF IG subroutines as shown:

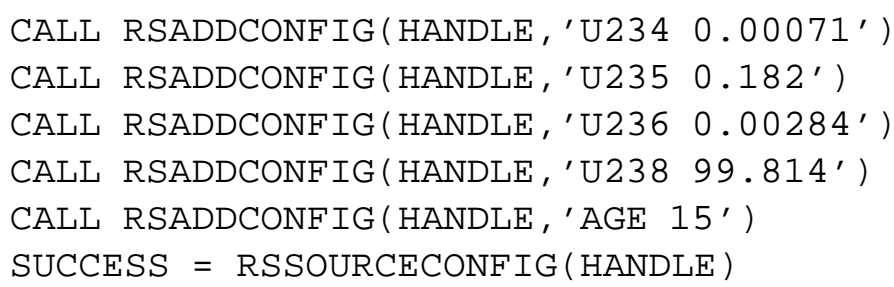

Finally, after the problem has been set up, the user can call the subroutine RSGTRPHOTON (HANDLE, RNG) to sample the photon distribution and return an energy value. The native random number generator is passed to the subroutine so that the Monte Carlo code can maintain control over the random number sequence. The subroutine returns an energy value in $\mathrm{keV}$.

\section{Configuring COG to use RADSRC}

The user will need to have access a COG installation.

Download the RADSRC library to your computer. It can be found at http://nuclear.llnl.gov. In the src directory type gmake in order to create the libradsrc.a file. It is highly recommended that you determine what compiler the COG installation is using and modify the RADSRC Makefile to use the same compiler.

COG provides the capability to compile a user source routine and dynamically link it into the COG executable. Make a directory containing the makefile COGUserlib.make and the user source subroutine I soP.F which can be found in the usrdet directory of your COG release. The makefile must be modified to link in the RADSRC library. Modify the LDOPTS variable to add:

LDOPTS $=\ldots$-L\$ (RADSRC_HOME)/lib/ .. -lradsrc -lstdc++

This is correct for the intel compiler. Other compilers may require different libraries. The environment variable RADSRC_HOME should be set to point to your installation of RADSRC. As the RADSRC library is written in $\mathrm{C}++$ one must also link in the stdc++ library.

At this point you can try to link the COG user library to ensure that the libraries are being properly linked. In order for the RADSRC library to be able to find its data files an environment variable must be set: 
setenv RADSRC_LEGACYDATA \$ (RADSRC_HOME)/data/

\section{Options Unique to COG}

The IsoP.F subroutine can also be configured to accept input from the COG input file. Using the code fragment:

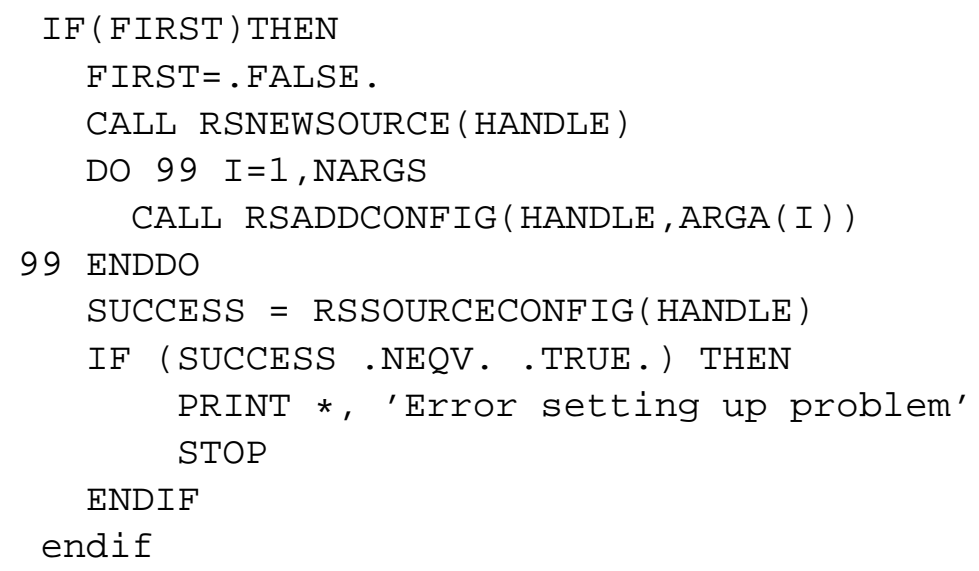

the COG user source input will be passed to the RADSRC library. Setup up the source specification in the COG input file with the following format:

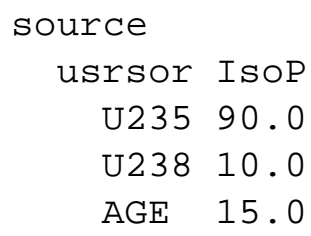

Due to the limitations of the COG input parser none of the individual words in the input can be longer than 8 characters.

\section{Configuring MCNP to use RADSRC}

The user will need to have access to and be able to recompile the MCNP source code.

Download the RADSRC library to your computer. It can be found at http://nuclear.llnl.gov. In the src directory type gmake in order to create the libradsrc.a file. It is highly recommended that you determine what compiler the MCNP installation is using and modify the RADSRC Makefile to use the same compiler.

The MCNP Makefiles must be modified to link in the RADSRC libraries. In the Source directory there is a master makefile and in the config directory there are platform specific makefiles. In the appropriate makefile for your installation add a line:

EXTRALIBS $=-1$ stdc ++ -L\$ (RADSRC_HOME) /lib/ -lradsrC

As the RADSRC library is written in $\mathrm{C}++$ one must also link in the stdc++ library. To allow the program to see the class definitions add a path to the $\mathrm{C}++$ headers by modifying

INCLUDE_DIRS = -I\$ (RADSRC_HOME)/src/libradsrc/ 
Ensure that the environment variable RADSRC_HOME points to your copy of the RADSRC installation. At this point you can try to relink the MCNP executable to ensure that the libraries are being properly linked. In order for the RADSRC library to be able to find its data files an environment variable must be set:

setenv RADSRC_LEGACYDATA \$ (RADSRC_HOME) / data/

\section{Future}

It is expected that this library and functionality will eventually be incorporated into MCNP and COG and will be directly callable from the input file. Please verify that your release of the Monte Carlo code does not have this functionality already in place. 